\title{
Innovación educativa en las prácticas de profesores de educación superior: aportes y beneficios
}

\author{
Rolando Guío Romero', \\ María Manuela Pintor Chávez², \\ Marcela Georgina Gómez Zermeño ${ }^{3}$
}

1. Fundación Universitaria Monserrate, (UNIMONSERRATE).Colombia; rolandoguio@hotmail.com 2. Instituto Tecnológico de Monterrey, (ITESM). México; maria.pintor@tecvirtual.mx 3. Instituto Tecnológico de Monterrey, (ITESM). México; marcela.gomez@itesm.mx

Recibido: 17 de abril del 2015

Corregido: 21 de julio del 2015

Aceptado: 03 de agosto del 2015

\begin{abstract}
Resumen
El presente artículo se desarrolla en torno a la temática de la innovación educativa y su relación con la labor docente de tres profesores en el nivel superior de una universidad colombiana. El estudio se realizó con una metodología cualitativa, recaudando la información mediante la entrevista a profundidad y la observación participante, para averiguar de qué manera se desarrolla la innovación educativa y conocer cuáles son sus percepciones sobre los retos y beneficios que impone dentro de su implementación en la práctica educativa. Entre los hallazgos, se encontró que los docentes reconocen la innovación como un elemento predominante en los modelos educativos actuales y se perfila como un valor agregado fundamental a la educación.
\end{abstract}

Palabras clave: innovación educacional, innovación pedagógica, educación superior, práctica pedagógica, universidad.

\section{Abstract \\ Educational innovation in higher education teaching practices: contributions and benefits}

This article presents a study about educational innovation in relation to the teaching practice in higher education. The investigation was developed with qualitative approach in a Colombian University with the participation of three professors, and we used in-depth interviews and participant observation to collect the data, that helped to find out how the teacher implement educational innovation in their contexts and hear what are their perceptions about the challenges and benefits of innovation. Among the findings, we found that teachers recognize educational innovation as a growing tendency in our current educational models, which is perceived as an added value in education. Keywords: educational innovations, teaching methods education, higher education, teaching practice, university.

Key words: educational innovation, educational innovation, higher education, teaching practice, university.

\section{INTRODUCCIÓN}

La necesidad de indagar sobre temas de interés educativo e identificar problemáticas destacadas en los procesos de aprendizaje es un ejercicio que promueve el presente trabajo de investigación. La innovación educativa forma parte de los modelos actuales de aprendizaje, es necesario que el docente genere nuevas prácticas educativas y que respondan a las necesidades actuales de los modelos educativos. El tema de innovación educativa permite reflexionar actualmente sobre dos aspectos, por un lado la exigencia de aprender a usar la tecnología y como segundo aspecto la manera como la tecnología puede ser un mecanismo y herramienta efectiva de conocimiento y comunicación. Es importante considerar que "en la medida que 
uno conozca más sobre lo que se puede hacer, sobre lo que se ha hecho, en esa medida es que podrá entrar al mundo de la innovación como eje transversal" (Ramírez, 2012, p.49).

Esta investigación tiene como objetivo: Identificar el nivel de conocimiento y empleabilidad que una muestra de profesores de educación superior poseen sobre el tema de innovación educativa a partir de sus prácticas y vivencias, determinando la efectividad y satisfacción de la implementación.

La pregunta que direccionó este estudio fue: ¿De qué manera se desarrolla la innovación educativa en las prácticas de profesores de educación superior y su apreciación frente a los aportes y beneficios? Entre los objetivos que se buscó cumplir a través del estudio, se encuentran:

1. Determinar las situaciones que se convierten en barreras en la implementación de innovaciones educativas.

2. Indagar la aceptación de la innovación educativa en docentes de educación superior.

3. Conocer las aportaciones que se consideran relevantes frente al tema de innovación educativa.

La investigación se llevó a cabo en la Universidad Minuto De Dios, sede Bogotá, Colombia. El estudio se desarrolló durante el año 2014, dividido en dos semestres. Se propuso analizar las diferentes perspectivas de los docentes frente a la temática del estudio, evidenciando cómo cada uno de ellos desarrolla estrategias innovadoras en los ambientes académicos y visualizando posibles barreras que dificulten la innovación desde la mirada de la academia. Cabe destacar que este estudio está orientado a conocer las condiciones y percepciones actuales, por lo cual esto es una primera etapa para los proyectos de innovación que la institución podría implementar en un futuro.

\section{Concepto de innovación}

La innovación educativa es un término complejo de delimitar, Macías (2005) define innovación como una mejora en los métodos, procesos, recursos, entre otros. Por lo tanto, el cambio ocurrido debe ser comprendido como una mejora. Entre sus propiedades, el autor enuncia que la innovación debe estar relacionada a una serie de elementos: la resolución de problemas, gestión democrática, cooperación, integralidad, descentralización y objetivos.

Para innovar no se trata de idealizar las prácticas, sino de enfrentar a la criticidad mediante la intervención académica y considerar modelos y alternativas en la educación que conduzcan a mejorar las prácticas que promuevan diversas maneras de adquirir e intercambiar experiencias. Rimari $(1996$, p. 5) menciona "una innovación no es buena por el hecho de ser nueva, sino básicamente porque contribuye de manera diferente, confiable y válida a solucionar problemas educativos o a mejorar quehaceres educativos".

La innovación convoca a las instituciones de educación superior a adquirir nuevas herramientas y talento humano, por tal motivo los docentes deben producir y proponer ideas de cambio en sus prácticas, teniendo en cuenta que "la innovación constituye la culminación de una serie de pasos relacionados con la formación, capacitación, motivación y fomento, pues instituye la convergencia entre la idea concebida y el producto terminado" (Alemán y Gómez-Zermeño, 2011, p.2).

Una innovación puede ser expresada por medio de estrategias, las cuales son entendidas como un conjunto de procedimientos con el propósito un objetivo definido, en este caso el aprendizaje (Rivero, GómezZermeño y Abrego, 2013). Es importante tomar en cuenta que en una innovación, el nivel del cambio propuesto puede ser el nivel de dificultades y resistencias que podrían emerger (De la Torre, 2009).

Para cambiar las dinámicas educativas, es necesario invitar a profesores a que consideren una nueva forma de realizar su labor de enseñanza, y es apropiado considerar que los cambios de paradigmas educativo demandan de procesos dinámicos (Carbajosa, 2011).

\section{- Innovación educativa en el nivel superior}

En la práctica universitaria, el docente debe impulsar la dinámica educativa del espacio académico de una manera propositiva. Los lineamientos de educación superior en Colombia se regulan por diversas entidades como el Ministerio de Educación Nacional (MEN) y por la Secretaría de Educación Distrital, las cuales realizan seguimiento a los procesos académicos con 
la finalidad de garantizar que los servicios educativos sean de calidad.

Una barrera en los procesos de innovación, es la inclusión y especialmente en cuanto al acceso a la tecnología. Las redes de internet facilitan el acercamiento a espacios que anteriormente eran muy difíciles de acceder por razones de tiempo, dinero, distancia, espacio, entre otros.

Actualmente, los modelos educativos innovadores tienen el reto de conservar los valores y buenas practicas académicas para que impacten positivamente en los actores educativos, considerando que "las crecientes demandas del contexto socioeconómico conducen a enfocarse a la necesidad de desarrollo de capital humano, campo en el que la educación adquiere gran relevancia" (Buendía y Martínez, 2007, p.81).

En países que se consideran en desarrollo, como el caso de Colombia, es fundamental reflexionar en nuevas prácticas académicas, en donde la educación es un medio para el fortalecimiento (Guerra, 2010).

De parte de los docentes de educación superior, el tema las competencias y su desarrollo es un asunto de continuo interés, por lo que es conveniente destacar que "el docente debe ser capaz de generar un progreso real de mejoramiento de aprendizajes significativos, acreditables, relevantes y autónomos (Salazar y Chiang, 2007, p. 26).

El documento sobre perspectivas tecnológicas para la educación superior en Iberoamérica 2012-2017 (Durall, Gros, Mainam Johnson y Adams, 2012), enuncia una serie de retos que van acorde con las tendencias actuales y orientados a la innovación educativa en este nivel. Entre los retos clave se encuentran: la transformación de las estructuras institucionales bajo un modelo de la sociedad del conocimiento, incorporación de la tecnología en la docencia e investigación, experiencias de aprendizaje abierto, competitividad y sustentabilidad de las instituciones educativas.

\section{METODOLOGÍA}

En el trabajo de investigación se utilizó el método cualitativo, el cual plantea una estructura flexible y constructiva que ofreció a los participantes e investigador la posibilidad de analizar varios factores desde la perspectiva personal. De acuerdo con Valenzuela y
Flores (2012), los investigadores cualitativos están interesados en cómo la gente interpreta sus experiencias, y cómo se construye el significado en el entorno de los sujetos estudiados.

Para este estudio, se seleccionó una muestra de manera intencional, teniendo tres docentes de educación superior pertenecientes a la Facultad de Ciencias Humanas y Sociales, del programa de Trabajo Social de una Universidad de la capital de Colombia.

Los instrumentos de investigación para la recolección de información fueron la entrevista a profundidad y la observación participante. La entrevista a profundidad permite una comunicación e interacción permanente y no necesariamente estricta y rígida entre el investigador y los participantes, de esta manera fue posible obtener mayor información y desarrollar los ejercicios en espacios abiertos que permitieron la recolección por parte de los tres docentes de manera natural y espontánea.

Por entrevista a profundidad, según Taylor y Bogdan (1990), se entiende a los encuentros entre el investigador y los sujetos de estudio, mediante una interacción constante que aporta sobre el tema en relación al desarrollo de sus vidas, experiencias y situaciones cotidianas. La entrevista se conformó de 20 preguntas abiertas que brindaron libertad de opiniones e indagación. La información se recabó de manera confidencial y para ser utilizada con fines académicos, orientada al tema de la implementación de innovaciones educativas y estrategias innovadoras en las prácticas de profesores de educación superior de este estudio:

1. ¿Para usted que significa el término de innovación educativa?

2. ¿Cuál uso específico ha dado a la innovación educativa en su ejercicio como docente?

3. ¿Conoce algunas herramientas que sean parte de los procesos innovadores a nivel educativo?

4. ¿Ha aplicado alguna o algunas herramientas que considere innovadoras en el desarrollo de sus clases?

5. Una vez que ya ha aplicado las estrategias innovadoras en sus cursos, ¿podría dar sus cursos sin implementar dichas estrategias? 
6. ¿Combina en sus cursos estrategias tradicionales con estrategias innovadoras?

7. ¿Qué alternativas ofrece la academia en la implementación de innovaciones educativas en sus prácticas?

8. ¿Qué rol desempeñan los estudiantes en la implementación de estrategias innovadoras?

9. ¿Cómo docente ha compartido la experiencia de la implementación de innovaciones educativas en sus cursos con otros docentes?

10. ¿Cuál ha sido la reacción de otros docentes al escuchar sobre la implementación de estrategias innovadoras en sus cursos?

11. ¿Qué representa para usted el emplear la innovación educativa?

12. ¿Considera que la innovación educativa genera aportes y beneficios en los actores académicos?

13. ¿Considera que existe alguna desventaja de implementar estrategias innovadoras en los procesos académicos?

14. ¿De qué forma considera que el uso de la tecnología forma parte de la innovación educativa?

15. ¿Considera que las estrategias innovadoras a nivel educativo pueden ser utilizadas para construir conocimiento?

16. ¿Cómo considera que se da la interacción entre el estudiante, el docente y las estrategias innovadoras?

17. ¿En el diseño de sus cursos se estipula la innovación educativa como parámetro o guía?

18. ¿En el diseño del curso existen actividades que realizan los estudiantes interactuando con tecnología y que a su vez proyecten innovación?

19. ¿Cómo relaciona la aplicación de estrategias innovadoras con los objetivos del curso?

20. ¿Recomendaría o recomienda a sus colegas la innovación educativa como estrategia para alcanzar objetivos académicos y mejorar las prácticas educativas?

La observación participante, según Bisquerra (2004), está orientada a "la descripción de grupos sociales y escenas culturales a través de la vivencia de las experiencias de las personas implicadas con el fin de captar como definen su propia realidad y los constructos que organizan su mundo" (p. 302). La observación se realizó en tres momentos durante las clases de cada uno de los docentes, lo cual permitió al investigador interactuar con el entorno y la realidad de estudio.

TABLA 1

Guía de observación

Criterios orientadores

Estrategias empleadas en la dinámica de la clase

Herramientas utilizadas en la dinámica del desarrollo de la clase

Estrategias innovadoras evidenciadas

Barreras observadas

Fortalezas observadas

Áreas de oportunidad observadas

Otras observaciones

Fuente: elaboración propia

Se aplicaron los dos instrumentos durante el trascurso del primer semestre del año 2014 a un docente que contribuyó para la prueba piloto, donde se validaron los instrumentos. La aplicación del instrumento se realizó posterior a la elaboración y firma de documento de consentimiento para los participantes y directivos de la facultad, establecimiento de fecha y diálogo con los participantes sobre la temática e investigación.

Para el análisis de la información, se eligió la estrategia de la triangulación la cual permite analizar los datos de cada una de los instrumentos y ser confrontado con el sustento teórico (Mathison, 1998).

Se contactó a cada uno de los participantes con la finalidad de obtener formalmente la carta de consentimiento que cuenta con la fecha en que se realizó el trámite, la firma y tentativamente el número de identificación, se concertaron las fechas y horas en que se dio la aplicación a cada uno de los instrumentos.

\section{RESULTADOS}

Para el análisis de la información recolectada de la entrevista, se fijaron cinco categorías. Las categorías 1, 
2 y 3 se relacionan con el primer objetivo de investigación: determinar las situaciones que se convierten en barreras durante la implementación de innovaciones educativas. La categoría 4 se liga al segundo objetivo, acerca de indagar la aceptación de una innovación educativa en docentes de educación superior; es decir, se busca recuperar información sobre la experiencia del docente. La categoría 5 corresponde al tercer objetivo, al buscar conocer las aportaciones relevantes frente al tema de innovación educativa.

De este modo, se pretendió comparar la teoría con las aportaciones de los tres docentes participantes. Respecto a las características de los participantes, se contó con la colaboración de tres profesoras con 5 , 9 y 10 años de experiencia docente, respectivamente. Entre sus similitudes, se encuentran entre los 30 y 40 años de edad, además tienen el grado de maestría. Entre sus diferencias, cada una indica tener una estrategia de aprendizaje preferida, como el aprendizaje basado en casos, seminario alemán, aprendizaje cooperativo y la discusión.

La tabla 2 presenta los resultados de la observación en clase con los profesores, de acuerdo con los criterios que se establecieron, para así analizar de cuál manera implementan la innovación en sus procesos de enseñanza.

TABLA 2

Resultados de la observación participante

Criterios observados Docente 1 Docente 2 Docente 3

Dinámica en clase

Herramientas utilizadas

en clase

Documentos escritos e impresos pizarra.

Llamado a lista, hora estricta de llegada a clase 10 minutos, discusión abierta y cuestionarios.

Ubicación en mesa redonda, documentos previos a clase, reflexión y control de lectura. derno de notas, lecturas extras y

Uso de tablero como apoyo y guía de la clase, presentación previa de la dinámica de clase, control de tiempos para cada actividad desarrollada, documento de clase brindado con anticipación y control de lectura.

Documento impreso, pizarra, cronómetro. cronometro.

\section{Estrategias o recursos utilizados}

Uso de equipos tecnológicos en clase para apoyar la dinámica, celular, computador audio, mapa conceptual, análisis de casos de la vida cotidiana, historias de vida.

\section{Dificultades al utilizar esas estrategias/recursos}

Posición magistral en clase, condicionamientos docente- estudiante en tiempos y métodos de evaluación, estudiantes con diferencia de semestres.
Afinidad y aceptación docente con el grupo y, flexibilidad en la búsqueda de materiales de apoyo al tema de clase, dominio del docente frente al tema, tono de voz y vocalización.

\section{Áreas de oportunidad} Manejo de equipos tecnológicos, experiencia en el ejercicio de la profesión, afinidad docente- alumnos.
Seminario alemán, dinámica activa entre participantes, el docente desempeña diferentes roles en su clase, uso de plataforma virtual y redes sociales como medio de comunicación.

Espacio reducido en el aula de clase, estudiantes con diferencia de más de 4 semestres, participación desigual del grupo.

Afinidad docente con el grupo, dominio del docente frente al tema de clase, acercamiento al estudiante, capacidad para acomodar el espacio y propiciar participación, tono de voz y vocalización.

Manejo de plataformas virtuales y redes sociales, afinidad docente- alumnos.
Estudio de casos, grupo de discusión, propiciar participación y valorar los aportes de estudiante, apoyo por aulas virtuales, foros, documentos digitales y REA.

Ubicación del docente de manera magistral, no se logra captar la atención total del grupo y su participación.

Afinidad docente con el grupo, dominio del docente frente al tema de clase, capacidad de explicación para facilitar comprensión, tono de voz y vocalización.

Manejo de tecnología y aulas virtuales. 
A continuación se presentan las categorías obtenidas de las respuestas de la entrevista con los profesores, en conjunto con lo obtenido de la observación (Tabla 2).

\section{Categoría 1: Ambiente de aula en contextos innovadores}

Se encontró que los tres docentes poseen como uno de los principales objetivos reconocer a sus estudiantes como sujetos activos. Según lo recaudado en la entrevista, manifestaron:

- Docente 1: "Las estrategias innovadoras sirven y son un canal efectivo de comunicación del docente con el estudiante en las dinámicas de clase".

- Docente 2: "Pienso que las dinámicas e innovación educativa generan conocimiento entre sus actores".

- Docente 3: "En mis clases intento que el estudiante sea un sujeto activo, en donde se entienda como un legítimo otro y se le dé la palabra para que tenga la oportunidad de discutir".

De acuerdo con los recursos y estrategias que se observaron en la práctica docente, se encontró el ambiente en el aula como un factor dominante en la práctica del docente que apunte a genere entornos innovadores, lo cual está en concordancia con lo que menciona Salazar y Chiang (2007) en referencia a que el docente debe ser capaz de generar un progreso de aprendizajes significativos, acreditables, relevantes y autónomos.

\section{Categoría 2: Postura del alumno frente a métodos innovadores}

En esta categoría se analizó la perspectiva de los alumnos y la manera en que se relacionan en entornos donde los docentes implementan o aplican estrategias innovadoras con la finalidad de promover su participación o construcción colectiva del conocimiento en la dinámica de enseñanza y aprendizaje, los diferentes roles que asumen y las actitudes que fueron observadas por los profesores en la aplicación de los instrumentos y estrategias innovadoras en clase.
En primera medida, es necesario mencionar los diferentes aspectos observados en la dinámica de clase:

- Docente 1: maneja un curso de 25 estudiantes y se implementó una metodología innovadora como el hecho de permitir que el estudiante interactúa con la tecnología y la internet para generar aportes al grupo, mapa conceptual estructurando ideas claves de la temática de clase, análisis de casos de la vida cotidiana e historias de vida.

- Docente 2: su clase estuvo compuesta por 28 estudiantes y se evidenció que utiliza estrategias como la aplicación del seminario Alemán, juego de roles e implementó una estructura en la ubicación de los participantes fuera de la cotidianidad y el orden jerárquico.

- Docente 3: su entorno estaba conformado por 29 estudiantes y en sus dinámicas de clase utilizó el grupo de discusión, estudio de casos y construcción de estructuras simbólicas sobre los aportes que realizaban los estudiantes. En este caso existía una limitante de ubicación que no permitía al docente implementar estructura diferente de él y sus estudiantes en el aula de clase.

En los tres casos, los estudiantes en su mayoría fueron receptivos, debido al hecho de que pudieron participar de la clase y sus aportes fueron tomados en cuenta para la construcción de los temas. Se logró captar la atención y los motivó para participar en una dinámica activa, a pesar de no contar en la mayoría de las clases con apoyos tecnológicos. Menciona Martínez (2013) que la enseñanza es un singular proceso de comunicación que no puede quedar al margen de las transformaciones que la tecnología incorpora a la sociedad.

\section{Categoría 3: Dinámica de enseñanza y aprendizaje}

Los resultados de la investigación permiten observar un claro reconocimiento a la labor del estudiante en la implementación de innovaciones educativas, anexo también es evidente que la labor del docente posee un impacto de respeto y liderazgo, eso permite observar que para implementar innovaciones educativas, es necesaria la participación activa de los actores académicos en un sentido integral. 
En este caso, los tres docentes participantes reconocieron en el proceso de enseñanza y aprendizaje la labor del estudiante como actor fundamental y apuestan a las prácticas innovadoras no desde lo nuevo, sino en el ejercicio de construir conocimiento compartido con el estudiante el cual trascienda a mejorar las prácticas en el reconocimiento del otro y la dinámica activa.

- Docente 1: "Es claro que las estrategias innovadoras sirven de canal entre el docente y el estudiante y que es necesario que la dinámica de enseñanza y aprendizaje sea de manera integral reconociendo sus diferentes actores".

- Docente 2: “En mi caso pasan de ser un estudiante que viene a aprender a un estudiante que viene a enseñar desde su experiencia, desde su vida, desde lo que saben, desde lo que leen, no los veo como alguien a quien les tengo que enseñar si no sujetos con quien tengo que reflexionar".

- Docente 3: "La relación educador-educando es el centro del proceso educativo, los dos tiene un rol igualmente importante que se construye a partir de una relación de carácter dialectico, para que existan los estudiantes deben existir los docentes y viceversa, en el desarrollo de clase deben actuar como pares".

Lo anterior concuerda con lo expresado por Rimari (1996) quien establece que no debe confundirse innovación con algo que es nuevo, sino porque contribuye de manera diferente, confiable y válida a solucionar problemas o mejorar quehaceres educativos.

La dinámica de enseñanza y aprendizaje está permeada por la innovación educativa, por proyectar prácticas novedosas en los docentes y sobre todo porque estas sean aprovechadas al máximo en la dinámica de enseñar y aprender, de los tres docentes podemos decir que comprenden la importancia de la innovación educativa en sus prácticas cotidianas, y aunque aún empleen herramientas consideradas como tradicionales como la pizarra y los marcadores en caso de los tres docentes, la proyección va más allá de únicamente registrar datos o trasmitir conocimiento.

- Docente 1: "Trato siempre de que una clase no sea igual a otra, por eso mi metodología es diferente y propicia innovación".

- Docente 2: "De ahí que ahora me acerco al aula no pensando en que voy a enseñar y aprender sino con quien lo voy a hacer, no pensando en enseñar para que otros aprendan, sino también aprendiendo a enseñar".

- Docente 3: "En mi ejercicio docente siempre me he preocupado en no repetir la lógica tradicional en donde el docente trasmite conocimiento al estudiante de manera mecánica, lógica que no permite el debate, que observa a los estudiantes como objetos de conocimiento y no como sujetos".

De acuerdo con la información obtenida en la entrevista y la observación, los participantes tienen claro que el tema de innovar en la educación y en los procesos de enseñanza y aprendizaje exige preparación y práctica, lo cual se puede constatar en lo mencionado por Ramírez (2012) quien afirma que la práctica docente involucra actitud, compromiso, valores y, sobre todo, apertura a la innovación como estrategia del aseguramiento de la calidad.

\section{- Categoría 4: Nivel de conocimiento y empleabilidad}

Es necesario resaltar en el caso del docente 1 y concerniente a esta categoría, que relaciona la innovación educativa directamente con las TIC, por lo cual sus respuesta son aisladas y en momentos se perciben barreras frente a la claridad en el tema de innovación educativa, esto en contradicción a lo que se observa en su clase en donde emplea estrategias innovadoras como permitir a sus estudiantes el uso de computadoras, celulares, asignarles roles, elaboración de mapas conceptuales, incentiva la construcción colectiva a partir de experiencias cotidianas, entre otras, lo que denota que, a pesar de tener un desempeño que apunta a lo innovador, no lo identifica claramente por el desconocimiento en el tema "considero que el uso de internet y uso de plataformas virtuales son algunas herramientas innovadoras".

Se observa en la respuesta que docente 1 ofrece en el instrumento que asocia el tema de la innovación educativa con el uso del internet y las TIC. Es necesario aclarar que a pesar de esta postura, durante la observación realizada el docente empleó múltiples herramientas y estrategias que son consideradas innovadoras y promueven un ejercicio académico dinámico, lo cual se relaciona con lo expuesto por Alemán y 
Gómez-Zermeño (2011) quienes aseveran que la innovación constituye la culminación de una serie de pasos relacionados con la formación, capacitación, motivación y fomento, es la convergencia entre la idea concebida y el producto terminado.

El nivel de conocimiento y empleabilidad de los docentes 2 y 3 podría manifestarse en límites de un buen manejo sobre el tema, argumentando que necesariamente lo nuevo no es lo innovador, los participantes comentan:

- Docente 2: "No es únicamente lo nuevo es la forma de hacer algo recogiendo lo que históricamente ha sucedido pero con un giro, con ajustes con miras a que genere impactos distintos".

- Docente 3: "Significa aplicar estrategias que rompan con la perspectiva tradicional de la educación, de manera que se promuevan escenarios de construcción colectiva, para incentivar la producción de conocimiento desde análisis compartidos y construidos de la realidad social".

En el caso de los tres docentes, ellos implementan diversas estrategias innovadoras las cuales fueron observadas en el desarrollo de sus clases, principalmente se tratan de dinámicas activas con sus grupos de estudiantes; entre ellas se observaron las mesas de discusión abierta, apoyo de aulas virtuales, ejemplos sobre vivencias cotidianas, seminarios, cambio de roles, recursos educativos abiertos, entre otros.

\section{- Categoría 5: Efectividad y satisfacción}

La efectividad de las estrategias utilizadas se evidenció en el ejercicio del docente en donde cada uno de los tres profesores emplea diversas estrategias que permiten ver un sello particular e individual en el ejercicio de la docencia y se detectó un alto grado de compromiso por la labor realizada, la misma que se proyecta no únicamente a las consignas teóricas, sino también a nivel personal, familiar, social y profesional que evidencian una comprensión clara por parte del estudiante y le permite argumentar su discurso y expresar los contenidos asimilados. Además, se observó el reconocimiento que el docente tiene por el estudiante como un sujeto activo dentro de su proceso estudiantil.
Cabe señalar que a pesar de las particularidades metodológicas de los docentes participantes, en todos se percibe la implementación de modelos que rompen los paradigmas tradicionales de la educación y permiten transformar la pasividad del estudiante para construir una dialéctica entre estudiante- docente, lo que confirma en este caso lo expuesto por Carbajosa (2011) quien afirma que "los paradigmas, en realidad, no tienen fronteras. Se trata más bien de tradiciones académicas".

En el instrumento de observación, cabe destacar en el docente 1 que su práctica acaparó la atención del grupo al emplear la estrategia de historias de vida con la que se planteó una construcción conjunta de grupo que permite considerar un modelo efectivo ya que la participación de los estudiantes en las clases observadas fue clara y contundente.

En el caso del docente 2, la observación permitió conocer que a pesar de las limitaciones estructurales del aula se sortea la situación con propuestas innovadoras en cuanto a la ubicación de los estudiantes, propiciando un ambiente dispuesto y con mayores comodidades para trabajar; además, se implementa como estrategia central del curso la técnica de seminario alemán en donde los alumnos y el docente asumen diversos roles que propician la participación de la mayoría del grupo y las retroalimentaciones que permite considerar que hay satisfacción y efectividad en el grupo.

El docente 3 en el instrumento de observación permite analizar diferentes factores como su dominio en el grupo empelando como estrategia central la construcción colectiva entre docente-estudiante, se hacen válidos cada uno de los aportes y se cuestiona en grupo de manera armónica y propositiva cada uno de los aportes, propiciando nuevas visiones que concluyen en un concepto final plasmado en un mapa conceptual, el cual es el producto final de la dinámica.

Lo anterior permite evidenciar lo que ratifica Ramírez (2012) quien menciona que para mejorar los resultados, se realizan cambios y constante seguimientos a dichas modificaciones.

Por tanto, se considera que la evaluación de resultados en esta categoría fue satisfactoria y se encontraron hallazgos importantes, se observa la preocupación de los docentes por asumir nuevos roles que conduzcan necesariamente a interactuar con el estudiante, reconocer sus potencialidades y habilidades, hacerlo participe de manera dinámica en el proceso académico 
y sobre todo cada una de las practica se tornó placentera para docentes y estudiantes.

\section{CONCLUSIONES}

A la luz de los datos obtenidos en la investigación se puede afirmar que el objetivo general se cumplió ya que se logró identificar que efectivamente existe conocimiento por parte de los docentes participantes sobre estrategias que propicien la innovación educativa en sus contextos. Por tanto, dicho conocimiento es reflejado en las prácticas y el desarrollo de clases, y existe una previa planeación que proyecta ejercicios innovadores pero que a su vez no es una proyección rígida ya que los profesores reconocen la posibilidad de generar cambios que se proponen en el ejercicio académico mancomunadamente entre estudiantes y docente.

Se debe destacar que específicamente el docente 1 ofrece una visión un poco sesgada de lo que es la innovación educativa ya que la relaciona específicamente al campo de las TIC y el uso del internet, aunque es necesario mencionar que no por esta razón sus clases no son innovadoras.

En relación a la efectividad y satisfacción de dichas prácticas, se puede hallar que tanto en docentes como en estudiantes es evidente la aceptación de la innovación educativa, se aprecia como el docente motiva al estudiante mediante metodologías que dinamizan su ejercicio, invitándolo a realizar construcciones colectivas que contribuyen a perder el temor de expresar lo que siente y a ejercer un rol activo.

Por tanto, se determina que la efectividad y satisfacción de la innovación educativa en la presente investigación es favorable, se refleja en la actitud de los actores y logra captar la atención de los estudiantes que anteriormente no experimentaban con prácticas novedosas, según afirman los profesores por las experiencias vividas con sus alumnos

El objetivo general se cumplió ya que se logró recaudar los datos necesarios que arrojaron como resultado un mayor nivel de conocimiento en los docentes 2 y 3 acerca del tema de innovación educativa. Por su parte, el nivel de empleabilidad de los tres participantes es favorable, lo que puede ser ratificado mediante lo observado en las prácticas y argumentos de estudiantes y docentes que permiten percibir satisfacción en el desarrollo de sus clases.

Respecto al objetivo: Determinar las situaciones que se convierten en barreras en la implementación de innovaciones educativas, se detectó que en primer lugar y particularmente con el docente, la barrera más importante es el desconocimiento frente a lo que es la innovación educativa ya que sesga dicha definición directa únicamente al uso de la tecnología y el internet, desconociendo otros componentes importantes y fundamentales del término, esto ocasiona que limite la posibilidad de fortalecer aún más sus prácticas y reconocer que su buena pedagogía educativa forma parte de los modelos innovadores y que es posible cada día generar nuevos aportes apoyados en lo que realmente significa el termino central de investigación.

Por otro lado, existe la barrera de los espacios físicos e infraestructura que determinan las universidades para el desarrollo de clases, se concluye que no existe una efectiva planeación en la asignación de las aulas para los cursos por lo cual existen espacios muy reducidos para cerca de 30 participantes y sillas ancladas al piso que necesariamente obligan al docente a recurrir, en gran medida, al modelo de clase magistral y uso del tablero. Dicha situación no permite que los actores implementen una ubicación determinada orientada a enriquecer sus prácticas y propiciar mayor participación o empleabilidad de estrategias como foros, mesas redondas, seminarios, entre otros.

Se considera también como barrera la manera en cómo se encuentran organizados los estudiantes en los diferentes cursos y aunque pertenecen al mismo pregrado existen diferencias en relación con el nivel de formación académica ya que algunos pertenecen a primeros semestres y otros se encuentran próximos a su graduación, lo cual provoca que el docente deba realizar mayores esfuerzos para propiciar la participación manera equitativa, que deba intervenir en la conformación de grupos de trabajo para propiciar equidad y que retome varias aportaciones para brindar mayor explicación.

En cuanto a indagar la aceptación que posee la innovación educativa en docentes de educación superior, es posible afirmar que los participantes consideran la innovación educativa como un tema posesionado de manera relevante en los modelos educativos y capaz de generar aportes fundamentales en educación, 
especialmente en educación superior, reconociendo claramente la importancia de su implementación en las dinámicas de enseñar y aprender bajo estrategias académica vanguardistas.

Los participantes poseen la conciencia de que la innovación educativa se encuentra inmersa en la educación superior y, más que una decisión de involucrarse o no en dichos modelos, significa estar en disposición para aportar a los actuales modelos mediante un ejercicio que demuestra ser efectivo y se ratifica en lo observado en las prácticas.

Es posible identificar que la aceptación que poseen los docentes es muy buena en virtud de lo que ellos afirman en sus testimonios y lo observado en sus prácticas, siendo evidente el placer por el ejercicio de su labor y la implementación de metodologías innovadoras, las cuales son efectivamente aceptadas por el grupo de estudiantes, de esta manera se permite afirmar que en el presente ejercicio los docentes reconocen y aceptan la innovación educativa como metodología vanguardista.

La información obtenida permitió conocer las aportaciones que se consideran relevantes en docentes frente al tema de innovación educativa, principalmente enfocados en dinamizar las metodologías y en el reconocimiento por la labor del estudiante frente a los procesos constructivos de enseñar y aprender.

Los participantes aportan actitud favorable y constructiva frente a los modelos educativos innovadores, realizan de manera constante el esfuerzo por estar a la vanguardia frente al tema y ofrecer a los estudiantes un ambiente diferente al acostumbrado de monotonía magistral en las clases, reconociendo que los modelos educativos vanguardistas deben ser construidos a partir de sustentos teóricos y prácticos.

Lo anteriormente expuesto permitió dar respuesta a la pregunta de investigación: ¿De qué manera se desarrolla la innovación educativa en las prácticas de profesores de educación superior y su apreciación frente a los aportes y beneficios? La implementación se realiza de manera teórico-práctica en construcción colectiva entre los actores educativos. Por tanto, el modelo de innovación educativa forma parte de la dinámica actual de aprendizaje y permite implementar de manera flexible estrategias para fortalecer los espacios académicos, reconociendo la diferencia y particularidades de cada entorno y sus actores.
El análisis de datos permitió concluir que la actitud del docente es importante en la dinámica educativa ya que permite contagiar al estudiante y en la medida que se realice la labor con agrado y dedicación, permitirá establecer compromiso entre educando-educador.

La educación es el principio de la solución para generar cambios sociales significativos. La temática central de investigación permite generar diferentes reflexiones, entre ellas el posicionamiento que toman los modelos educativos innovadores mediante el planteamiento de estrategias y competencias entre los actores del proceso educativo.

Se recomienda en futuras investigaciones ampliar el campo de investigación y abarcar a los diferentes actores educativos puesto que cada uno de ellos se encuentra condicionado en cierta medida por el otro. También es necesario comprender y analizar las pretensiones de cada uno de ellos en relación con la innovación y sus expectativas en la mejora de la calidad de vida y la calidad educativa.

\section{REFERENCIAS}

Alemán, L., Gómez-Zermeño, M. G. (2012). Liderazgo Docente para la Enseñanza de la Innovación. Revista de Investigación Educativa de la Escuela de Graduados en Educación, 2(4), 2-7. Recuperado de: http://rieege.tecvirtual.mx/index.php/rieege/article/view/76

Bisquerra, R. (2004). Metodología de la investigación educativa. Madrid: La Muralla

Buendía, A. y Martínez, A. (2007). Hacia una nueva sociedad del conocimiento: retos y desafíos para la educación virtual. En A. Lozano Rodríguez y J. V. Burgos Aguilar (Ed.), Tecnología educativa en un modelo de educación a distancia centrado en la persona (pp. 77-106). México: Limusa.

Carbajosa, D. (2011). Debate desde paradigmas en la evaluación educativa. Perfiles Educativos, 33, 183-192.

De La Torre, S. (2009). La universidad que queremos. Estrategias creativas en el aula universitaria. Revista Digital Universitaria, 10(12), 1-17.

Duarte, J. M. (2008). El valor de las TIC en educación superior. RUSC. Universities and Knowledge Society Journal, 5(1).

Durall, E., Gros, B., Maina, M., Johnson, L. \& Adams, S. (2012). Perspectivas tecnológicas: educación superior en Iberoamérica 2012-2017. Austin, Texas: The New Media Consortium. 
Freire, P. (1970). Pedagogía del Oprimido. Montevideo: Tierra Nueva.

Guerra, D. (2010). La descentralización educativa: estrategia para el desarrollo. México: Instituto Politécnico Nacional.

Macías, A. B. (2005). Una conceptualización comprehensiva de la innovación educativa. Innovación educativa, 5(28), 19-31.

Martínez, E. (2013). Una mirada hacia la inclusión: barreras en el camino a la participación. Perspectiva Educacional, Formación de Profesores, 52(2), 177-200.

Mathison, S. (1988). Why Triangulate? Educational Researcher. Washington, DC: AERA

Ramírez, M. (2012). Modelos y estrategias de enseñanza para ambientes innovadores. Monterrey, México: Editorial Digital del Tecnológico de Monterrey.
Rimari, W. (1996). La innovación educativa: un instrumento de desarrollo. Recuperado de: http://www.uaa.mx/direcciones/dgdp/defaa/descargas/innovacion_educativa_octubre.pdf

Rivero, I., Gómez-Zermeño, M.G., y Abrego, R. (2013). Tecnologías educativas y estrategias didácticas: criterios de selección. Revista electrónica Educación y Tecnología, 3. Recuperado de http://revistas.umce.cl/edytec/article/ view/134

Salazar, C. M. y Chiang, M. (2007). Competencias y educación superior. Un estudio empírico. Horizontes Educacionales, 12(2), 23-35.

Taylor, S. y Bogdan, R., (1990). Introducción a los métodos cualitativos de investigación. Buenos Aires, Argentina: Paidós.

Valenzuela, J. y Flores, M. (2012). Fundamentos de la Investigación Educativa. Monterrey, México: Editorial Digital del Tecnológico de Monterrey. 
